\title{
Versatile MOF-derived cobalt catalyst for the reductive amination
}

\author{
Haihong $\mathrm{Wu}^{1,2}$, Chaoren Shen ${ }^{1}$, Chungu $\mathrm{Xia}^{{ }^{1 *}}$ and $\mathrm{Lin} \mathrm{He}^{\mathrm{1}^{*}}$
}

The tunable metal center and the tailorable organic linker of metal-organic framework (MOF) make the characteristic of ultrahigh porosity, controllable pore size, crystalline nature and highly-ordered structures [1]. Since its emergence $[2,3]$, it represents one of the most celebrated functional materials. Owing to the rich diversity of MOF family, a vast of important applications including the storage of gas, recognition of molecules as well as environment remediation have been developed [1,4]. Moreover, the extensive utilization of MOF as heterogeneous catalyst [5] and host matrices for immobilization of molecular catalysts [6] has demonstrated its versatility in applied catalysis.

Meanwhile on the other aspect, as one of the well-established electrocatalysts for the oxygen reduction reaction [7], transition-metal nanoparticles (NPs) supported on nitrogen-doped carbon were groundbreakingly introduced into the liquid-phase catalytic hydrogenation and oxidation of organic molecules by the research group of Prof. Matthias Beller in Leibniz-Institut für Katalyse [8-10]. The nitrogen atoms implanted by pyrolyzing metal-phenanthroline complexes immobilized on support contributed to the catalytic reactivity of metal. Since then, these fascinating materials have enabled a series of progress for many important catalytic transformations $[11,12]$. Some insightful understandings about the effect of nitrogen atoms and metal cluster on catalytic performance have been revealed as well $[13,14]$. However, most of these catalysts exhibit poor reactivity and selectivity, challenging multicomponent synthetic organic reactions.

To address this issue, Beller and co-workers developed MOF-derived cobalt NP catalyst supported on nitrogendoped carbon by taking full advantage of uniform topology and strong bonds between inorganic and organic units in MOF [15]. The nitrogen-contained cobalt MOF comprising two organic linkers 1,4-diazabicyclo[2.2.2] octane (DABCO) and terephthalic acid (TPA) was employed as structure directing template. The pyrolysis of in-situ assembled cobalt-DABCO-TPA MOF supported on carbon at $800^{\circ} \mathrm{C}$ under argon produced graphitic shell encapsulated cobalt nanoparticles (Fig. 1), which is the most active material. Characterization disclosed that there

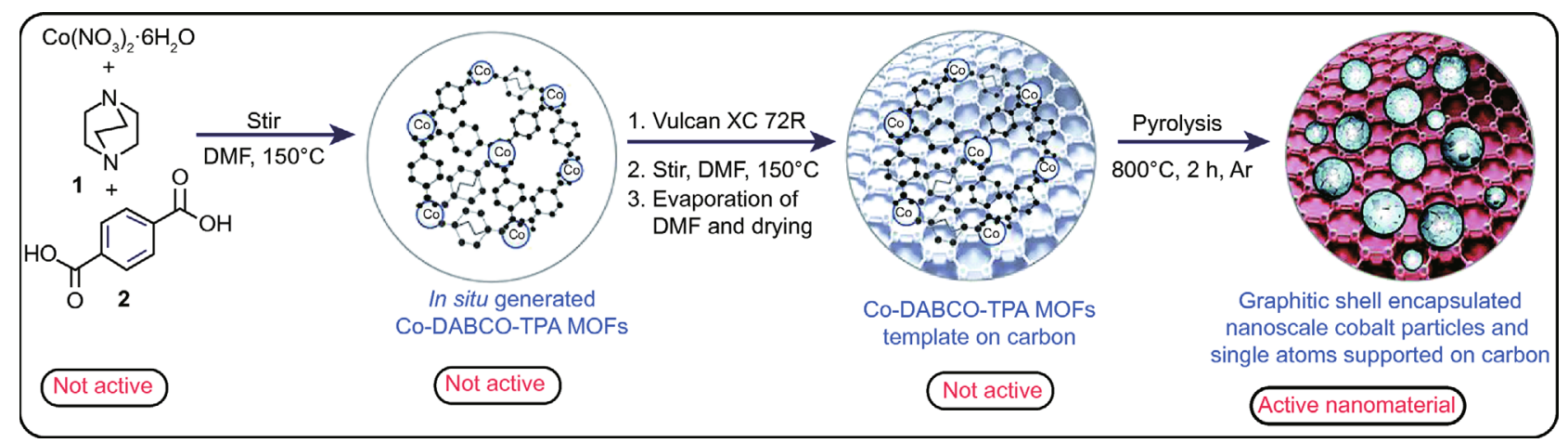

Figure 1 Preparation of graphitic shell encapsulated cobalt nanoparticles supported on carbon using MOF precursors. Reprinted with permission from Ref. [10], Copyright 2017, American Association for the Advancement of Science.

\footnotetext{
${ }^{1}$ State Key Laboratory for Oxo Synthesis and Selective Oxidation, Suzhou Research Institute of LICP, Lanzhou Institute of Chemical Physics (LICP), Chinese Academy of Sciences, Lanzhou 730000, China

${ }^{2}$ University of Chinese Academy of Sciences, Beijing 100049, China

*Emails: cgxia@licp.cas.cn (C Xia), helin@licp.cas.cn (L He)
} 
are two major types of cobalt species in this MOF-derived catalyst: the metallic cobalt particles surrounded by a combination of some graphic layers and single Co atoms in the short-range ordered graphic shells. High angle annular dark field images of $\mathrm{C} / \mathrm{N}$ overlay unveil that nitrogen is located not only in the graphitic shell on the Co particle but also in short range ordered carbon which contains single Co atoms. Cobalt traces are detectable everywhere in the nitrogen containing carbon at low concentration. Both of DABCO and TPA have strong effect on the valence and morphology of cobalt species as well as the state and quantity of nitrogen in the nearsurface region of material after pyrolysis. Neither material generated in the absence of either organic linker is reactive as the cobalt catalyst derived from cobalt-DABCOTPA MOF. Furthermore, pyrolyzing temperature is critical for both structural formation of graphitic shells enveloping the metallic Co, which is claimed to be vital for high activity and stability. Note that cobalt is unique for the title reaction; materials derived from $\mathrm{Fe}-, \mathrm{Mn}-, \mathrm{Cu}-$ and Ni-DABCO-TPA showed no or very weak activity.

The superb performance of heterogeneous cobalt catalyst derived from MOF in reductive amination of aldehyde and ketone to primary, secondary and tertiary amines (149 examples) is impressve (Fig. 2). In the reductive amination of aldehydes and ketones with the atmosphere of ammonia and hydrogen to give linear and branched primary amines (Fig. 2a), the heterogeneous Co catalyst exhibits excellent chemoselectivity and tolerance to sensitive functional groups such as halides, esters, carbon-carbon multiple bonds, heteroaromatic rings and sulfur-containing groups. The undesired subsequent formation of secondary and tertiary amines, which was usually overcome by means of noble metal catalysts or flammable Raney Ni, can be avoided over this MOF-derived Co catalyst. The reductive amination of aliphatic aldehydes that often hindered by unproductive aldol condensation also proceeds very well. Bio-active phenethylamine, potent central nervous system stimulating drug amphetamine and its analogs were prepared in good yield. The stability and recyclability of this heterogeneous Co catalyst is validated with the results of recycling catalyst six times without any significant loss of catalytic activity during the synthesis of amphetamine. Moreover, the protocol was successfully applied into the late-stage modification of three natural products, three non-steroidal anti-inflammatory agents as well as five steroids. The scales for synthesizing some of these products were able to be enlarged up to 1000 times without decline on yield. By switching the amine source from $\mathrm{NH}_{3}$ to pri- a Co-DABCO-TPA@C-800 catalyzed reductive amination of aldehydes and ketones

$$
\mathrm{R} \curvearrowright \mathrm{O} \frac{\text { Co-DABCO-TPA@C-800 (3.5 mol.\% Co) }}{\mathrm{NH}_{3} \text { (5-7 bar), } \mathrm{H}_{2} \text { (40 bar), } t \text { - } \mathrm{BuOH}, 120^{\circ} \mathrm{C}} \mathrm{R} \widehat{\mathrm{NH}_{2}}
$$$$
41 \text { examples, } 80 \% \sim 92 \% \text { yield }
$$

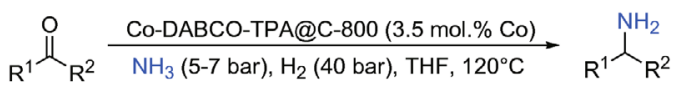

46 examples, $77 \% \sim 92 \%$ yield

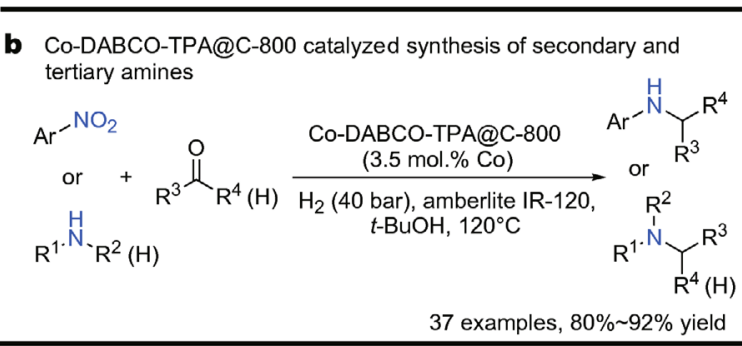

C Co-DABCO-TPA@C-800 catalyzed preparation of $\mathrm{N}$-methylamines

$$
\begin{aligned}
& \mathrm{R}_{\mathrm{O}}+\underset{\mathrm{N}}{\frac{\mathrm{H}}{\mathrm{H}_{2}(40 \mathrm{bar}), \mathrm{t}-\mathrm{BuOH}, 120^{\circ} \mathrm{C}} \mathrm{R}_{\mathrm{N}}^{\text {Co-DABCO-TPA@C-800 }}} \\
& 13 \text { examples, } 79 \% \text { 92\% yield } \\
& \mathrm{R}^{-\mathrm{NO}_{2}}+\mathrm{HCHO}_{\text {or }} \frac{\text { Co-DABCO-TPA@C-800 }}{\mathrm{H}_{2}(40 \mathrm{bar}), t-\mathrm{BuOH}, 120^{\circ} \mathrm{C}} \mathrm{R}_{-} \\
& \mathrm{R}^{-\mathrm{NH}_{2}} 12 \text { examples, } 80 \% \sim 90 \% \text { yield }
\end{aligned}
$$

Figure 2 Synthesis of primary, secondary and tertiary amines catalyzed by MOF-derived Co catalyst. The MOF-derived Co catalyst is denoted as Co-DABCO-TPA@C-800.

mary or secondary amines, the corresponding secondary or tertiary amines are able to be produced in good yield and selectivity without any over-alkylation products (Fig. 2b). This merit on chemoselectivity is sufficiently presented in the $\mathrm{N}$-alkylated derivatives of amino acid esters and preparation of ten existing drug molecules containing the moiety of secondary or tertiary amines (Fig. 3). This pathway avoids precious metal-based catalysts and waste from sodium borohydride reductant in previous methods. In the reductive amination of aldehydes to secondary arylamines by directly employing nitroarene as the surrogate of aniline, the multiple functionality of this MOFderived Co catalyst is displayed on simultaneously undertaking two different types of reduction. Besides these, the Co catalyst is also effective to the direct reductive methylation of nitroarenes or amines with aqueous formaldehyde to the corresponding $\mathrm{N}$-methylamines (Fig. 2c). $N$-dimethylated derivative of natural product Salicin, antidepressant Venlafaxine and $\mathrm{N}$-monomethylated derivative of antidepressant Paroxetine can be obtained with this method in high yields.

In conclusion, Beller et al. exemplified how to inter- 


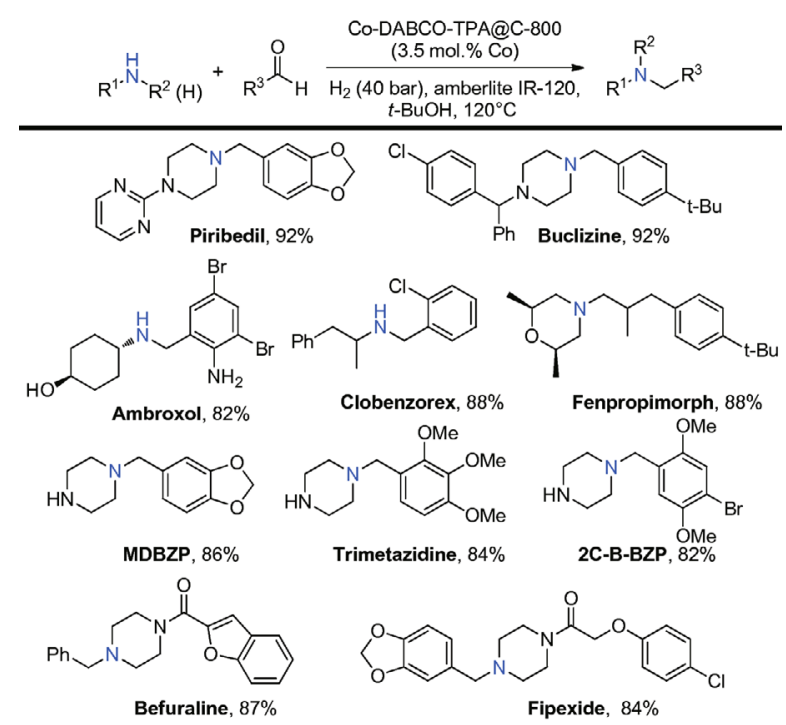

Figure 3 The synthesis of ten existing drug molecules by Co-catalyzed reductive amination of aldehydes with primary or secondary amines.

disciplinarily explore the versatile applications of simple MOF material in applied heterogeneous catalysis for the synthesis of primary, secondary, tertiary amines including a big group of useful molecules. It will be expected that this nice piece of work will stimulate more intercommunication and cooperation between the research area of material synthesis and applied catalysis and promote the research on applying novel materials to advanced heterogeneous catalysis.

Received 12 October 2017; accepted 12 October 2017; published online 28 November 2017

1 Kaskel S (ed.). The Chemistry of Metal-Organic Frameworks: Synthesis, Characterization, and Applications. Weinheim: Wiley$\mathrm{VCH}, 2016$

2 Chui SSY, Lo SMF, Charmant JPH, et al. A chemically functio- nalizable nanoporous material $\left[\mathrm{Cu}_{3}(\mathrm{TMA})_{2}\left(\mathrm{H}_{2} \mathrm{O}\right)_{3}\right]_{n}$. Science, 1999, 283: $1148-1150$

3 Li H, Eddaoudi M, O'Keeffe M, et al. Design and synthesis of an exceptionally stable and highly porous metal-organic framework. Nature, 1999, 402: 276-279

4 Schröder M (ed.). Functional Metal-organic Frameworks: Gas Storage, Separation and Catalysis. Berlin-Heidelberg: Springer, 2012

5 Huang YB, Liang J, Wang XS, et al. Multifunctional metal-organic framework catalysts: synergistic catalysis and tandem reactions. Chem Soc Rev, 2017, 46: 126-157

$6 \mathrm{Wu} \mathrm{CD}$, Zhao M. Incorporation of molecular catalysts in metalorganic frameworks for highly efficient heterogeneous catalysis. Adv Mater, 2017, 29: 1605446

7 Wood KN, O'Hayre R, Pylypenko S. Recent progress on nitrogen/ carbon structures designed for use in energy and sustainability applications. Energ Environ Sci, 2014, 7: 1212-1249

8 Jagadeesh RV, Wienhöfer G, Westerhaus FA, et al. Efficient and highly selective iron-catalyzed reduction of nitroarenes. Chem Commun, 2011, 47: 10972-10974

9 Jagadeesh RV, Surkus AE, Junge $\mathrm{H}$, et al. Nanoscale $\mathrm{Fe}_{2} \mathrm{O}_{3}$-based catalysts for selective hydrogenation of nitroarenes to anilines. Science, 2013, 342: 1073-1076

10 Jagadeesh RV, Junge H, Beller M. Green synthesis of nitriles using non-noble metal oxides-based nanocatalysts. Nature Comm, 2014, 5: 4123

11 He L, Weniger F, Neumann H, et al. Synthesis, characterization, and application of metal nanoparticles supported on nitrogendoped carbon: catalysis beyond electrochemistry. Angew Chem Int Ed, 2016, 55: 12582-12594

12 Li M, Xu F, Li H, et al. Nitrogen-doped porous carbon materials: promising catalysts or catalyst supports for heterogeneous hydrogenation and oxidation. Catal Sci Technol, 2016, 6: 3670-3693

13 Liu W, Zhang L, Liu X, et al. Discriminating catalytically active $\mathrm{FeN}_{x}$ species of atomically dispersed $\mathrm{Fe}-\mathrm{N}-\mathrm{C}$ catalyst for selective oxidation of the C-H bond. J Am Chem Soc, 2017, 139: 1079010798

14 Ji S, Chen Y, Fu Q, et al. Confined pyrolysis within metal-organic frameworks to form uniform $\mathrm{Ru}_{3}$ clusters for efficient oxidation of alcohols. J Am Chem Soc, 2017, 139: 9795-9798

15 Jagadeesh RV, Murugesan K, Alshammari AS, et al. MOF-derived cobalt nanoparticles catalyze a general synthesis of amines. Science, 2017, 358: 326-332 